\title{
Update on the effect of age on acute pancreatitis morbidity: a retrospective, single-center study
}

\author{
Banu Kara ${ }^{1}$, Sehmus Olmez ${ }^{1}$, Mehmet Suat Yalıın², Adnan Tas ${ }^{1}$, Nevin A. Ozturk ${ }^{1}$, Bunyamin Sarıtaş ${ }^{3}$ \\ ${ }^{1}$ Department of Gastroenterology, Health Sciences University, Adana, Turkey \\ 2Department of Gastroenterology, Education and Research Hospital, Aksaray University, Aksaray, Turkey \\ ${ }^{3}$ Department of Gastroenterology, Sanlıurfa Mehmet Akif Inan Education and Research Hospital, Sanlıurfa, Turkey
}

Gastroenterology Rev 2018; 13 (3): 223-227

DOI: https://doi.org/10.5114/pg.2018.75677

Key words: acute pancreatitis, prognosis, geriatric population, mortality.

Address for correspondence: Assoc. Prof. Banu Kara, Department of Gastroenterology, Health Sciences University, Adana City Hospital, 01060 Adana, Turkey, phone: +90 5056177309, e-mail: banu.banu97@gmail.com

\begin{abstract}
Introduction: Acute pancreatitis (AP) is one of the urgent diseases of gastroenterology. Due to the growth of the elderly population, the frequency of the disease in the elderly population is also increasing.

Aim: To evaluate the contributing factors of mortality in geriatric patients (age $\geq 65$ years) and non-geriatric (age $<65$ years) patients.

Material and methods: We retrospectively analyzed data of consecutive patients with AP, in the Adana Numune Education and Research Hospital between March 2013 and September 2015.

Results: Of the 602 patients studied, 405 were female and 197 were male and their mean age was $55.2 \pm 19.5$ years. The most common etiological factors were biliary stone, hyperlipidemia and alcohol, respectively. Two hundred and four patients were in the geriatric group and 394 patients were in the non-geriatric group. $84.4 \%$ of patients had mild AP, and $15.6 \%$ of patients had moderate to severe AP according to the revised Atlanta classification. $91.7 \%$ of non-geriatric patients had mild AP while $70.7 \%$ of geriatric patients had mild AP $(p<0.001)$. $29.4 \%$ of geriatric patients had moderate-to-severe AP while $8.4 \%$ of non-geriatric patients had moderate-severe AP. Duration of hospital stay was $6.2 \pm 3$ days and $5.3 \pm 2.3$ days in geriatric and non-geriatric groups respectively $(p<0.001)$. Mortality was higher in the geriatric group than the non-geriatric group $(9.6 \%$ vs. $0.5 \%$, respectively) $(p<0.001)$.

Conclusions: Acute pancreatitis in the geriatric population shows a more severe course than the non-geriatric population. Geriatric patients have longer duration of hospital stay and higher mortality than non-geriatric patients.
\end{abstract}

\section{Introduction}

Acute pancreatitis (AP) is an inflammatory disease of the pancreas with various clinical courses. Acute pancreatitis prevalence is increasing in the world [1]. Eighty percent of patients have mild acute pancreatitis, which is self-limiting and heals completely without serious complications in a couple of days, while $20 \%$ of patients have severe pancreatitis [1]. Although mortality in AP is decreasing and it is $2 \%$ at present, it is higher in elderly patients [1, 2].

It is important for clinicians to evaluate the severity of AP, to determine the need for intensive care unit admission, prognosis and management [3-5]. As the elderly population increasing, it is important to determine the prognosis of AP in the geriatric population.
There are many scoring systems and laboratory parameters to determine the prognosis [3-6]. Ranson Criteria, Modified Glasgow Score, Acute Physiology and Chronic Health Evaluation II (APACHE II) are widely used scores. There are some limitations of these scoring systems. The recently revised Atlanta classification is used widely. Patients with AP are classified into mild, moderate and severe AP according to the revised Atlanta classification [7].

Although it is thought that elderly patients with AP had a worse clinical course, there are only a few studies comparing disease course according to age. There are conflicting results on the effect of AP on mortality in the geriatric population. While some studies reported that mortality is unchanged in geriatric patients, some 
other studies reported higher mortality rates in geriatric patients $[8,9]$.

\section{Aim}

Thus, we aimed to evaluate mortality and influencing factors in geriatric and non-geriatric patients with AP.

\section{Material and methods}

We retrospectively analyzed data of patients with acute pancreatitis, in the Adana Numune Education and Research Hospital between March 2013 and September 2015. Acute pancreatitis diagnosis was established by two of the following three features: 1 ) characteristic abdominal pain; 2) increased amylase and lipase levels (> 3 times the upper limit of normal); 3 ) imaging consistent with AP [1]. Patients' age, gender, etiology of AP, laboratory parameters, Ranson score, revised Atlanta classification, duration of hospital stay and mortality rates were recorded. The revised Atlanta classification (mild: no organ failure and no local or systemic complications; moderately severe: transient organ failure $(<48 \mathrm{~h})$ and/or local or systemic complications without persistent organ failure (> $48 \mathrm{~h}$ ); severe: persistent organ failure (> $48 \mathrm{~h}$ ): single organ failure or multiple organ failures) was used to diagnose the severity of AP [6].

Patients were grouped into two groups: the geriatric ( $\geq 65$ years) and non-geriatric group ( $<65$ years). We compared both groups according to gender, etiology of AP, laboratory parameters, Ranson criteria, revised Atlanta classification, duration of hospital stay and mortality rates.

All patients underwent abdominal imaging (ultrasonography and/or computed tomography according to the severity). The etiology was defined as biliary if gallstones or sludge was found. Alcoholic AP was considered if the patient had consumed more than $40 \mathrm{~g}$ of ethanol per day ( $20 \mathrm{~g}$ in female) for at least 5 years or if the patient had consumed an excessive amount of alcohol shortly before the onset of the disease (more than five standard drinks/60 g) [8]. A serum triglyceride level more than $1000 \mathrm{mg} / \mathrm{dl}$ and exclusion of other etiologies were accepted as a hyperlipidemic etiology [8]. We excluded patients who had chronic pancreatitis with acute relapse.

\section{Statistical analysis}

The statistical significance level was set at 0.05 . All analyses were performed using MedCalc Statistical Software version 12.7.7 (MedCalc Software bvba, Ostend, Belgium; http://www.medcalc.org; 2013). Descriptive statistics were used to define continuous variables and mean, median, standard deviation, minimum, and maximum values were calculated. Comparison of two independent and normally distributed continuous variables was made with Student's $t$ test, and the comparison of two independent variables which do not fit the normal distribution was performed by the Mann-Whitney $U$ test. Relationships of categorical variables were analyzed by the $\chi^{2}$ test or Fisher's exact test.

\section{Results}

One thousand ninety-seven (32.75\%) male and 405 (67.3\%) female patients were included in the study. The median age was $55.2 \pm 19.5$ years old. Two hundred and eight $(34.6 \%)$ patients were in the geriatric group and 394 (65.4\%) patients were in the non-geriatric group. Mean ages were $76.9 \pm 7.7$ years in the geriatric group and $43.8 \pm 13$ years in the non-geriatric group. The most frequently observed etiologic factors were biliary stone disease, hypertriglyceridemia, and alcohol (81.6\%). Patients' demographic, etiologic factors, laboratory parameters, scoring system results, duration of hospital stay and mortality rates are shown in Table I.

In the geriatric group 147 (70.7\%) patients had mild AP, 43 (20.7\%) patients had moderate AP and 18 (8.7\%) patients had severe AP according to the revised Atlanta classification. In the non-geriatric group 361 (91.7\%) patients had mild AP, 29 (7.4\%) patients had moderate AP and 4 (1\%) patients had severe AP according to the revised Atlanta criteria. There was a statistically significant difference between geriatric and non-geriatric groups using the revised Atlanta classification $(p<0.001)$ (Table I). The Ranson score was $2.1 \pm 1.2$ in the geriatric group and $1.1 \pm 1.1$ in the non-geriatric group and there was a statistically significant difference between groups ( $p<0.001)$. Duration of hospital stay was $6.2 \pm 3$ days and $5.3 \pm 2.3$ days in geriatric and non-geriatric groups, respectively. The geriatric group had a statistically significantly longer duration of hospital stay $(p<0.001)$. Mortality rates were $9.6 \%$ in the geriatric group and $0.5 \%$ in the non-geriatric group and there was statistically significant difference $(p<0.001)$ (Table I). In the non-geriatric group 1 (3.4\%) patient died in the moderate AP group, and 1 (25\%) patient died in the severe AP group. In the geriatric, moderate AP group $6(13 \%)$ patients died, in the severe AP group 14 (77\%) patients died. The patients who died in the geriatric, moderate AP group had the following co-morbid diseases: heart failure (3 patient), chronic obstructive pulmonary disease (2 patient), atherosclerotic heart disease (1 patient). The patient who died in the non-geriatric, moderate AP group had cirrhosis.

In the geriatric group creatinine values were significantly higher than the non-geriatric group. Albumin and 
Table I. Characteristics of patients with acute pancreatitis

\begin{tabular}{|c|c|c|c|c|}
\hline Parameter & $\begin{array}{l}\text { All patients } \\
(n=602)\end{array}$ & $\begin{array}{c}\text { Non-geriatric } \\
(<65 \text { years }) \\
(n=394)\end{array}$ & $\begin{array}{c}\text { Geriatric } \\
(\geq 65 \text { years }) \\
(n=208) \\
\end{array}$ & $P$-value \\
\hline Age [years] & $55.2 \pm 19.5$ & $43.8 \pm 13$ & $76.9 \pm 7.7$ & $<0.001^{2}$ \\
\hline \multicolumn{5}{|l|}{ Gender: } \\
\hline Female & $405(67.3 \%)$ & $244(61.9 \%)$ & $161(77.4 \%)$ & $<0.001^{1}$ \\
\hline Male & $197(32.7 \%)$ & $150(38.1 \%)$ & $47(22.6 \%)$ & \\
\hline \multicolumn{5}{|l|}{ Etiology: } \\
\hline Biliary & $428(71.1 \%)$ & $253(64.2 \%)$ & $175(84.1 \%)$ & \\
\hline Hypertriglyceridemia & $36(6 \%)$ & $34(8.6 \%)$ & $2(1 \%)$ & \\
\hline Alcohol & $27(4.5 \%)$ & $25(6.4 \%)$ & $2(1 \%)$ & $<0.001^{3}$ \\
\hline Drugs & $14(2.3 \%)$ & $8(2 \%)$ & $6(2.9 \%)$ & \\
\hline Cancer & $1(0.02)$ & $1(0.03 \%)$ & - & \\
\hline Idiopathic & $96(15.9)$ & $73(18.5 \%)$ & $23(11.1 \%)$ & \\
\hline Ranson score & $1.43 \pm 1.219$ & $1.1 \pm 1.1$ & $2.1 \pm 1.2$ & $<0.001^{2}$ \\
\hline \multicolumn{5}{|l|}{ Revised Atlanta Classification: } \\
\hline Mild & $508(84.4 \%)$ & $361(91.7 \%)$ & $147(70.7 \%)$ & \\
\hline Moderate & $72(12 \%)$ & $29(7.4 \%)$ & $43(20.7 \%)$ & $<0.001^{1}$ \\
\hline Severe & $22(3.6 \%)$ & $4(1 \%)$ & $18(8.7 \%)$ & \\
\hline WBC & $12398.6 \pm 4695$ & $12059.5 \pm 4218.5$ & $13042.5 \pm 5440.2$ & $0.055^{2}$ \\
\hline $\mathrm{Hb}$ & $13 \pm 1.8$ & $13.2 \pm 1.8$ & $12.5 \pm 1.7$ & $<0.001^{4}$ \\
\hline Glucose & $152 \pm 63.8$ & $150.2 \pm 67.5$ & $155.3 \pm 56.2$ & $0.004^{2}$ \\
\hline Albumin & $4.6 \pm 14.1$ & $4.9 \pm 17.3$ & $4.0 \pm 4.3$ & $<0.001^{2}$ \\
\hline AST & $193.1 \pm 232.9$ & $190.6 \pm 233.8$ & $197.8 \pm 231.5$ & $0.045^{2}$ \\
\hline ALT & $167.3 \pm 198.1$ & $185.3 \pm 223.3$ & $133.2 \pm 132.4$ & $0.352^{2}$ \\
\hline ALP & $156.5 \pm 119.9$ & $150.5 \pm 97.8$ & $167.7 \pm 152.1$ & $0.972^{2}$ \\
\hline GGT & $270.2 \pm 277.3$ & $286.4 \pm 287.7$ & $240.3 \pm 255.1$ & $0.124^{2}$ \\
\hline LDH & $393.2 \pm 254.7$ & $387.8 \pm 264.6$ & $403.2 \pm 235.4$ & $0.206^{2}$ \\
\hline Bilirubin & $2.2 \pm 12.6$ & $2.3 \pm 15.4$ & $2.0 \pm 3.7$ & $0.023^{2}$ \\
\hline Creatinine & $0.9 \pm 0.8$ & $0.8 \pm 0.9$ & $1 \pm 0.5$ & $<0.001^{2}$ \\
\hline CRP & $2.5 \pm 4.4$ & $2.2 \pm 4.2$ & $2.9 \pm 4.8$ & $0.650^{2}$ \\
\hline Duration of hospital stay [days] & $5.6 \pm 2.6$ & $5.3 \pm 2.3$ & $6.2 \pm 3$ & $<0.001^{2}$ \\
\hline \multicolumn{5}{|l|}{ Outcome: } \\
\hline Discharge & $580(96.3 \%)$ & $392(99.5 \%)$ & $188(90.4 \%)$ & $<0.001$ \\
\hline Exitus & $22(3.7 \%)$ & $2(0.5 \%)$ & $20(9.6 \%)$ & \\
\hline
\end{tabular}

WBC - white blood cells, Hb - hemoglobin, AST - aspartate aminotransferase, ALT - alanine aminotransferase, ALP - alkaline phosphates, GGT- $\gamma$-glutamyl

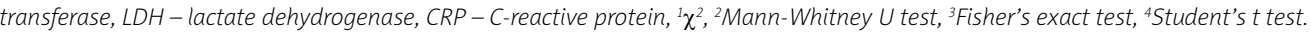


hemoglobin values were lower in the geriatric group than the non-geriatric group $(p<0.001)$ (Table I).

White blood cell counts, alanine aminotransferase, $\gamma$-glutamyl transpeptidase, alkaline phosphatase, lactate dehydrogenase and CRP values were not different between groups (Table I). Glucose, aspartate aminotransferase and bilirubin levels were significantly different between groups.

\section{Discussion}

The elderly population in the world is increasing and therefore the incidence of diseases in the elderly population is also increasing [10]. In most studies the mean age of the first AP attack is 60 years and in most western countries the incidence of AP has increased because of the increased elderly population [11]. With increasing age, the incidence and mortality of AP are also increasing, and there have been studies showing a correlation between age and mortality as an independent parameter [1, 12]. However, there are controversial results regarding the effect of age on disease severity and prognosis. Gardner et al. reported higher organ failure and three times higher mortality rates in geriatric patients in a study concerning 56 geriatric patients ( $\geq 70$ years old) and 56 non-geriatric controls $(<70$ years old) [13]. Uomo et al. reported that age and accompanying comorbidities had a limited effect on disease course and outcomes in a study involving 340 non-geriatric patients ( $<70$ years old) and 99 geriatric patients ( $\geq 70$ years old) (total 439 patients) with AP [14]. There are also studies reporting no effect of age on disease course in patients with AP. Kim et al. reported no significant influence of age on the clinical outcome of AP in a study involving 85 elderly ( $\geq 65$ years old) and 142 non-elderly ( $<65$ years old) patients with AP [8]. Similarly, Fan et al. reported no effect of age on mortality in patients with AP but assumed that concomitant medical and surgical problems might contribute to higher mortality rates in elderly patients [15]. In our study, we found mortality rates of $9.6 \%$ in geriatric patients and $0.5 \%$ in non-geriatric patients and there was a statistically significant difference $(p<0.001)$. Also in our study group, only $1 \%$ of non-geriatric patients had severe pancreatitis in accordance with studies indicating the importance of age on disease severity.

There are many scoring systems, imaging methods and laboratory parameters to determine the prognosis of AP. In clinical practice, it is important to predict the disease severity and need for intensive care unit admission, the duration of hospital stay and prognosis.

In severe pancreatitis on admission, the duration of hospital stay, need for ICU admission, and mortality would be higher than mild-moderate pancreatitis. Although it can be predicted that elderly patients might be hospitalized longer than non-elderly patients, Kim et al. reported longer duration of hospital stay in the non-elderly group than the elderly group in a study consisting of 227 patients with AP (11.9 \pm 10.1 days vs. 10.3 \pm 9.6 days in non-elderly and elderly patients, respectively) [8].

Xin et al. reported 21 days of hospital stay in geriatric patients and 19 days in non-geriatric patients, without a statistically significant difference [16]. In our study, we found $5.3 \pm 2.3$ days in the non-geriatric group and $6.2 \pm 3$ days in the geriatric group, with a statistically significant difference $(p<0.001)$.

The Atlanta classification and Ranson score are widely used to evaluate the severity of AP, and the Atlanta classification was revised in 2012 [7]. Losurdo et al. reported higher Ranson scores in geriatric patients than non-geriatric patients $(2.52 \pm 1.57$ vs. $0.75 \pm 0.73$ in geriatric and non-geriatric patients, respectively) in a study including 42 geriatric patients and 48 non-geriatric patients $(p<0.0001)$ [17]. Xin et al. also reported higher Ranson scores in geriatric patients than non-geriatric patients ( $3.4 \pm 1.7$ vs. $2.8 \pm 1.6$ in geriatric and non-geriatric patients, respectively) ( $p<0.0069)$ [16]. In our study, we found the Ranson scores to be $2.1 \pm 1.2$ in the geriatric group and $1.1 \pm 1.1$ in the non-geriatric group, with a statistically significant difference. Losurdo et al. reported mild pancreatitis in 39\% of patients and moderate-severe AP in $61 \%$ of patients according to the revised Atlanta classification [17]. In our study, $84.4 \%$ of patients had mild AP $(91.7 \%$ of patients in the non-geriatric group and $70.7 \%$ of patients in the geriatric group, $p<0.001)$. $8.4 \%$ of patients had moderate-severe AP patients in the non-geriatric group and $29.4 \%$ of patients in the geriatric group had moderate-severe $\operatorname{AP}(p<0.001)$.

There are limitations of the study. It is a retrospective study and all the patients' clinical monitoring processes were obtained from records. Since cases where sufficient records could not be obtained were not included in the study, the number of patients could not be as many as requested. The inadequate number of cases may have caused false positives in statistical values.

\section{Conclusions}

Geriatric patients had a more severe course in AP than the non-geriatric population. The geriatric population had a longer duration of hospital stay and a higher mortality rate than the non-geriatric population. When the elderly population increases and these results are evaluated together, we expect more serious acute pancreatitis in the near future. 


\section{Conflict of interest}

The authors declare no conflict of interest.

\section{References}

1. Forsmark CE, Swaroop Vege S, Wilcox CM. Acute pancreatitis. N Engl J Med 2016; 375: 1972-81.

2. Krishna SG, Hinton A, Oza V, et al. Morbid obesity is associated with adverse clinical outcomes in acute pancreatitis: a propensity-matched study. Am J Gastroenterol 2015; 110: 1608-19.

3. DengL, Wang L, Yong F, et al. Prediction of the severity of acute pancreatitis on admission by carboxypeptidase-B activation peptide: a systematic review and meta-analysis. Clin Biochem 2015; 48: 740-6.

4. Kuo DC, Rider AC, Estrada P, et al. Acute pancreatitis: what's the score? J Emerg Med 2015; 48: 762-70.

5. Cho JH, Kim TN, Chung HH, et al. Comparison of scoring systems in predicting the severity of acute pancreatitis. World J Gastroenterol 2015; 21: 2387-94.

6. Lipiński M, Rydzewska G. Immature granulocytes predict severe acute pancreatitis independently of systemic inflammatory response syndrome. Gastroenterology Rev 2017; 12: 140-4.

7. Banks PA, Bollen TL, Dervenis C, et al. Classification of acute pancreatitis - 2012: revision of the Atlanta classification and definitions by international consensus. Gut 2013; 62: 102-11.

8. Kim JE, Hwang JH, Lee SH, et al. The clinical outcome of elderly patients with acute pancreatitis is not different in spite of the different etiologies and severity. Arch Gerontol Geriatr 2012; 54: 256-60.

9. Lankisch PG, Burchard-Reckert S, Petersen M, et al. Etiology and age have only a limited influence on the course of acute pancreatitis. Pancreas 1996; 13: 344-9.

10. Beard JR, Officer A, de Carvalho IA, et al. The World report on ageing and health: a policy framework for healthy ageing. Lancet 2016; 387: 2145-54.

11. Spanier BW, Dijkgraaf MG, Bruno MJ. Epidemiology, aetiology and outcome of acute and chronic pancreatitis: an update. Best Pract Res Clin Gastroenterol 2008; 22: 45-63.

12. Goldacre MJ, Roberts SE. Hospital admission for acute pancreatitis in an English population, 1963-98: database study of incidence and mortality. BMJ 2004; 328: 1466-9.

13. Gardner TB, Vege SS, Chari ST, et al. The effect of age on hospital outcomes in severe acute pancreatitis. Pancreatology 2008; 8: 265-70.

14. Uomo G, Talamini G, Rabitti PG, et al. Influence of advanced age and related comorbidity on the course and outcome of acute pancreatitis. Ital J Gastroenterol Hepatol 1998; 30: 616-21.

15. Fan ST, Choi TK, Lai CS, et al. Influence of age on the mortality from acute pancreatitis. Br J Surg 1988; 75: 463-6.

16. Xin MJ, Chen H, Luo B, et al. Severe acute pancreatitis in the elderly: etiology and clinical characteristics. World J Gastroenterol 2008; 14: 2517-21.

17. Losurdo G, Iannone A, Principi M, et al. Acute pancreatitis in elderly patients: a retrospective evaluation at hospital admission. Eur J Intern Med 2016; 30: 88-93.

Received: 15.02 .2018

Accepted: 25.04 .2018 